This item was submitted to Loughborough's Research Repository by the author.

Items in Figshare are protected by copyright, with all rights reserved, unless otherwise indicated.

\title{
A novel orthogonal set adaptive line enhancer tuned with fourth-order cumulants
}

PLEASE CITE THE PUBLISHED VERSION

PUBLISHER

(c) IEEE

VERSION

VoR (Version of Record)

LICENCE

CC BY-NC-ND 4.0

\section{REPOSITORY RECORD}

Constantinides, A.G., K.M. Knill, and Jonathon Chambers. 2019. "A Novel Orthogonal Set Adaptive Line Enhancer Tuned with Fourth-order Cumulants”. figshare. https://hdl.handle.net/2134/5867. 
This item was submitted to Loughborough's Institutional Repository (https://dspace.lboro.ac.uk/) by the author and is made available under the following Creative Commons Licence conditions.

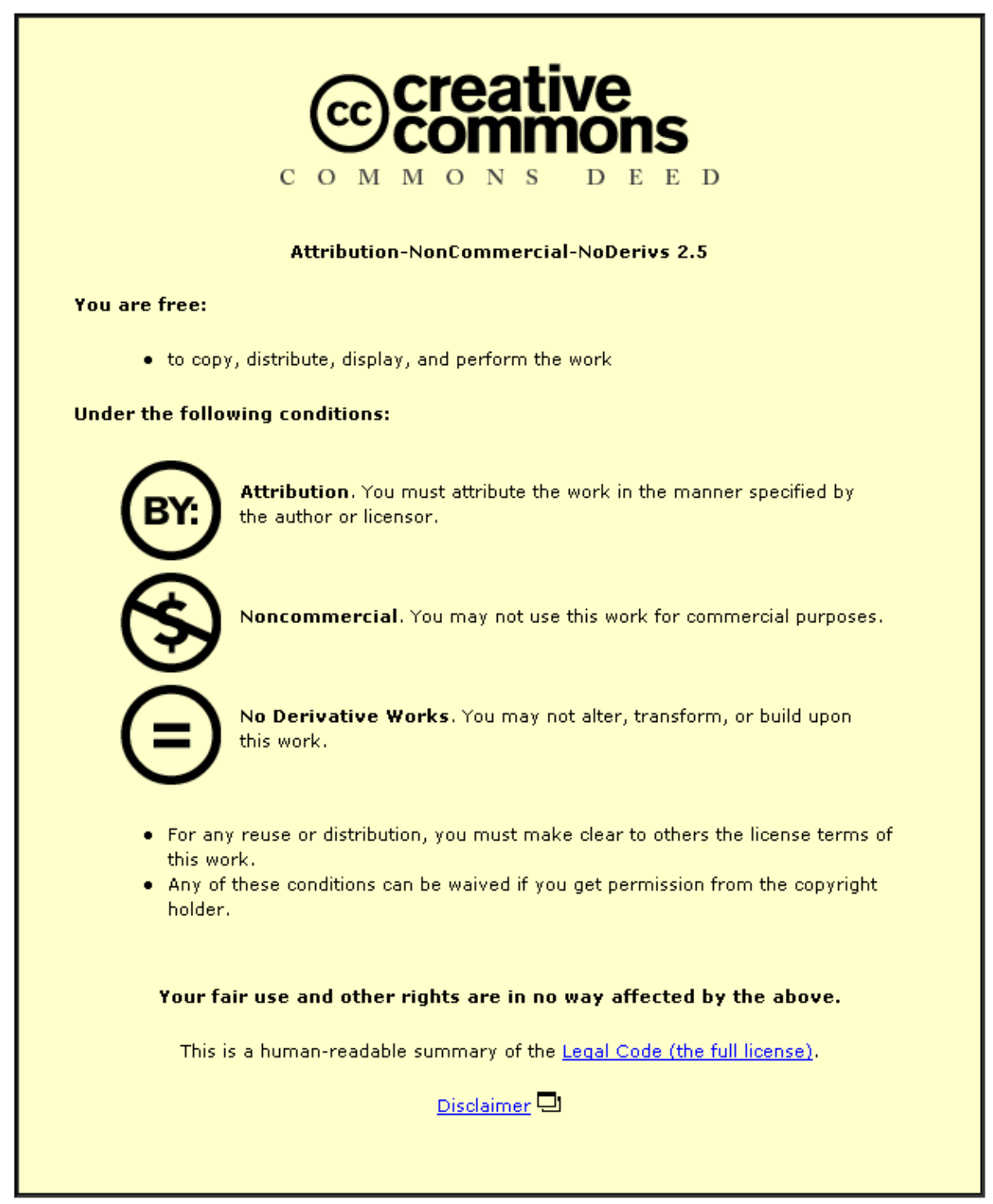

For the full text of this licence, please go to: http://creativecommons.org/licenses/by-nc-nd/2.5/ 


\title{
A NOVEL ORTHOGONAL SET ADAPTIVE LINE ENHANCER TUNED WITH FOURTH-ORDER CUMULANTS
}

\author{
A.G. Constantinides, K.M. Knill, and J.A. Chambers \\ Signal Processing Section, Department of Electrical and Electronic Engineering, \\ Imperial College, London, U.K.
}

\begin{abstract}
This paper introduces a novel adaptive line enhancer (ALE) structure. The adaptive filter has an autoregressive-moving average (ARMA) structure which is based on classical Laguerre orthogonal functions. The frequencies and radii of the poles within the orthogonal set admit tuning. Tuning of these terms in this contribution involves the use of higher order statistics. Indeed the properties of fourth order cumulants are exploited to aid harmonic retrieval of the sinusoids embedded in the input signal. Further. more adaptation of the feedforward filter parameters is straightforward since they are linearly related to the filter output. Advantages of stability, short filter length and robustness in the presence of noise are expected. Simulation results are included to show typical performance.
\end{abstract}

\section{INTRODUCTION}

The detection of sinusoids in noise is a recurrent problem in signal processing. When no a priori knowledge of the possibly time-varying sinusoidal parameters is available an adaptive solution is preferred. The first adaptive filtering approach was proposed by Widrow et al. [1] in a key-note paper in 1975. The operation of this technique, based on an Adaptive Line Enhancer (ALE), is effected by virtue of the predictability of the sinusoidal component(s) compared to the background noise. It has found many application areas which include sonar, biomedical and speech signal processing.

ALEs can be categorised by their structure, namely Finite Impulse Response (FIR) or Infinite Impulse Response (IIR). Initial development of the ALE centered on FIR filters $[2,3]$ because adaptation is straightforward. Unfortunately, such structures are frequently sub-optimal for modelling the underlying process. To achieve a given performance at low signal-to-noise ratio (SNR) increasingly long filter lengths are required. An IIR filter structure is an optimal solution [4-6], since significantly fewer param. eters are needed to achieve a desired performance. However, problems of instability and non-linearity arise due to the feedback in IIR filters. Additionally, the non-linear dependence of the error on the filter parameters can cause local minima in the error performance surface, which influences the system operation.

In this paper a novel ALE structure is proposed which has an ARMA form capable of producing a narrowband frequency response at low SNR combined with linearly related adaptive coefficients. Moreover, this new structure, the Orthogonal Block ALE (OBALE) employs fourth-order cumulants in its adaptation which is expected to improve performance when the input noise is zero mean Gaussian.

\section{PROPOSED ORTHOGONAL BLOCK ALE ARCHITECTURE \\ 2.1. Adaptive Fulter \\ 2.1.1. Structure}

The transfer function, $\mathrm{H}(\mathrm{z})$, of an FIR filter can be viewed as the linear combination of a set of functions, the delay elements $\left\{z^{-i}\right\}$, orthogonal around the unit circle. In the OBALE the function set $z^{-i}$ is replaced by a set, $G_{l}(z)$, which obeys the same rules of orthogonality [7]. The orthogonality of the set is important to ensure the optimum error in the least squares sense is achieved [8]. $\mathrm{H}(\mathrm{z})$ becomes the weighted sum of the individual components of the orthogonal set

$$
H(z)=\sum_{i=0}^{N-1} a_{i} G_{i}(z)
$$

The linearity between the coefficients $\left\{a_{i}\right\}$ and the function set $G_{i}(z)$ is retained irrespective of the individual nature of the components of $G_{i}(z)$. This allows each $G_{i}(z)$ "block" to be set up as an IIR filter without loss of the overall transversal structure Figure 1. Moreover, it implies that a simple adaptation algorithm such as Least Mean Squares (LMS) or Fast 
Least Squares (FLS) can be used to adapt the coefficients $\left\{a_{i}\right\}[9]$.

The chosen set for $G(z)$ can be any suitable orthogonal set preferably with complete properties. In this paper classical Laguerre functions have been chosen. Laguerre functions provide an advantage over other classical series such as Legendre functions in that both real and complex realisations of the filter are possible. These functions have previously been employed to good effect in transient analysis, network synthesis and most recently in adaptive echo cancellation $[10,11]$.

In the real case the individual set components are calculated using a procedure outlined by Broome [12]. $G_{0}(z)$ is chosen to be unity. The nth term in the set, $G_{n}(z), \mathrm{n}>1$ (n odd), is as follows

$$
\begin{aligned}
& G_{n}(z)=\frac{(z+1)}{\left(z-r_{n} e^{j \pi v_{n}}\right)\left(z-r_{n} e^{-\delta 2 \pi v_{n}}\right)} \\
& \times \prod_{i=1}^{n-1} \frac{\left(1-z r_{i} e^{j \pi v_{l}}\right)\left(1-z r_{i} e^{-\beta \pi v_{l}}\right)}{\left(z-r_{i} e^{j 2 \pi v_{l}}\right)\left(z-r_{i} e^{-\beta 2 \pi v_{l}}\right)}
\end{aligned}
$$

where $r_{n}$ is the radius and $\pm v_{n}$ the frequency of the nth complex conjugate pole pair. $G_{n}(z)$ can be seen to consist of two parts, a bandpass filter centered at frequency $v_{n}$, with a single zero at $z=-1$. and an allpass filter. The $(n+1)$ th term replaces the zero with one at +1 , but is otherwise identical.

Similarly we can form a complex 1st-order set of orthogonal function blocks. For the complex case the kth term in the set is as follows

$$
G_{k}(z)=\frac{1}{\left(z-c_{k}\right)} \prod_{i=1}^{k-1} \frac{\left(1-A_{i}(z)\right)}{\left(z-c_{i}\right)}
$$

where $G_{o}(z)$ is unity, $c_{k}=r_{k} e^{\Omega \pi v_{k}}$. The set of coefficients $\left\{c_{k}\right\}$ are therefore complex. When all the radii, $\left\{r_{k}\right\}$, are equal and all the frequencies, $\left\{v_{k}\right\}$, are set to zero the discrete Laguerre functions are obtained.

\subsubsection{Implementation}

The orthogonal block filter can be realised as a "lattice" filter type structure, similar to the expansion of Nie et al. of an orthonormal rational function[13]. In the real case each orthogonal block output, $G_{2 n+1}$, is related to the previous term, $G_{2 n-1}$, as follows

$$
\begin{gathered}
G_{2 n+1}(t)=G_{2 n-1}(t-2)-b_{n} G_{2 n-1}(t-1) \\
+r^{2}\left(G_{2 n-1}(t)-G_{2 n-1}(t-2)\right)+b_{n+1} G_{2 n-1}(t-1) \\
n=1,2,3 \ldots, p
\end{gathered}
$$

where $r$ is the fixed pole radius, $b_{n}=2 r \cos \left(2 \pi v_{n}\right)$ and $p$ the total number of complex pole pairs in the filter. The resulting structure is shown in Figure 2.

Note the same relationship exists between the $n$ even terms, i.e. $G_{2 n}, G_{2 n+2}$. Stability of the filter has been ensured by constraining the pole radii to lie on a fixed circle of radius less than but close to unity. A corresponding reduction in the number of adaptation calculations required is achieved.

For the complex case neighbouring terms are related as follows

$G_{k}(t)=G_{k-1}(t-1)-c_{k-1}^{*} G_{k-1}(t)+c_{k} G_{k}(t-1)$

This can be realised in a similar "lattice" type structure to the real case.

\subsection{Harmonic Retrieval of Sinusolds}

The performance of the OBALE is highly dependent on a good initial choice of the pole frequencies within the orthogonal blocks. To achieve this an initial estimate of the pole positions is formed through the cumulant-based approach to the harmonic retrieval problem of Swami and Mendel [14]. Closely related to Pisarenko's method [15], this method utilises fourth order statistics in place of Pisarenko's second order terms. It does so to take advantage of the cumulant properties of Gaussian noise. Above second order the cumulants of a Gaussian process are zero independent of whether the noise is white or coloured. The OBALE is required to operate at very low levels of SNR, below $-15 \mathrm{~dB}$, and so this feature is expected to aid pole selection. Fourth order cumulants are necessary as, in the absence of any phase coupling, the third order cumulant, $C_{x}^{3}(\tau)$, of a sum of sinusoids is zero.

Briefly the harmonic retrieval approach is as follows. Assume zero mean signals and that the input signal is given as

$$
y(t)=x(t)+w(t)
$$

where $x(t)$ is the sum of $p$ sinusoids

$$
x(t)=\sum_{i=1}^{p} a_{i} \cos \left(\omega_{i}+\phi_{i}\right)
$$

$\phi_{i}$ are iid random variables uniformly distributed over $(-\pi, \pi)$ and $w(t)$ is Gaussian noise. Estimate the fourth order cumulants of the input signal, $y(t)$, along a diagonal slice of the trispectrum. Typically all delays are chosen to equal $\tau$. Since $w(t)$ is Gaussian it's fourth order cumulant $C_{w}^{4}(\tau)$ is zero and thus

$$
C_{y}^{4}(\tau)=C_{x}^{4}(\tau)
$$


This leads to a set of linear equations of the form

$$
C_{y \underline{a}}=\underline{0}
$$

where $C_{y}$ is a Toeplitz cumulant matrix and $a$ is the eigenvector corresponding to it's zero eigenvalue. As with Pisarenko's method [15] the sinusoid frequencies are given by the roots of a polynomial $A(z)$ where $A(z)$ equals

$$
A(z)=\sum_{i=0}^{2 p} a_{i} z^{-i}
$$

If the additive noise is non-Gaussian, i.e. it has non-zero kurtosis, then the right hand side of equation 6 is replaced by $\gamma_{4} w$, the fourth order cumulant of the noise.

\subsection{Operation}

The OBALE operation consists of two parts as shown in Figure 3. Initially, the cumulant estimator runs alone. An estimate of the number and location of sinusoids within the input signal is made based on the fourth order cumulants of the input signal. The associated frequency estimates are fed to the orthogonal block coefficients $\left\{b_{i}\right\}$, and full operation commences.

As the OBALE runs the output error is passed to the adaptive algorithm. The Normalised Least Mean Squares (NLMS) algorithm [9] varies the weighting of the adaptive coefficients $\left\{a_{i}\right\}$. NLMS is chosen to overcome a change in signal variance due to the orthogonal set. At time $t$ the NLMS algorithm for the OBALE is

$$
\underline{a}(t)=\underline{a}(t-1)+\frac{\mu}{\hat{\sigma}_{g}^{2}(t)} \underline{g}(t-D) e(t)
$$

where $\mu$ is the adaptive gain constant, $g(t)$ the orthogonal block output vector, $e(t)$ the ALE output error and $\hat{\sigma}_{g}^{2}(t)$ an estimate of the signal power. The signal power is calculated as follows for each block output

$$
\hat{\sigma}_{g}^{2}(k, i)=\lambda \hat{\sigma}_{g}^{2}(k-1, i)+(1-\lambda)|g(k, i)|^{2}
$$

where $\lambda$ is a forgetting factor.

The ALE output is fedback into the cumulant estimator which is periodically checked to see if there is any improvement in the pole position estimate. If so, the revised estimate is fed to the coefficients $\left\{b_{i}\right\}$ and operation continues as before.

\section{SIMULATION RESULTS}

An example of the simulation results achieved with the OBALE is presented in Figure 4 and Figure 5.
The input to the ALE consists of a single sinusoid at normalised frequency $0.1 \mathrm{~Hz}$ in white Gaussian noise with a SNR of $-15 \mathrm{~dB}$. The ALE output spectrum is taken after 3000 iterations. A significant increase in the output SNR is observed even though the OBALE has not fully converged. The SNR at the OBALE output improves further as convergence is reached. The levels of improvement in SNR found with the OBALE compare with those of previous workers $[4,5]$. Its performance at low SNR is particularly promising. No stability problems are encountered with the linear adaptation.

\section{CONCLUSION}

A novel ALE structure has been presented which links orthogonal functions with fourth-order cumulants. The OBALE allows an IIR structure to be implemented and adapted by a simple algorithm, with no stability problems.

\section{ACKNOWLEDGEMENT}

The UK Science and Engineering Research Council and Marconi Underwater Systems Limited, U.K., provided funding for this work.

\section{References}

[1] B.Widrow et al.,"Adaptive Noise Cancelling: Principles and Applications", Proc.IEEE,v63,1975

[2] J.R.Treichler,"The Spectral Line Enhancer",Ph.D Thesis,Stanford Univ.,1977

[3] I.R.Zeidler et al.,"Adaptive Enhancement of Multiple Sinusoids in Uncorrelated Noise", IEEE Trans.ASSP,v26,1978

[4] B.Friedlander,"A Recursive Maximum Likelihood Algorithm for Adaptive Line Enhancement", IEEE Trans.ASSP, v30-4,1982

[5] M.El-Sharkaway and B.Peikai,"Adaptive Stochastic Filters with No Strict Positive Real Condition",IEEE Trans.ASSP,v35-11,1987

[6] C.R.Johnson,Jr.,"Adaptive IIR Filtering.Current Results and Open Issues", IEEE Trans.Info.Theory, v30,1984

[7] J.A.Chambers, "Digital Signal Processing Algorithms and Structures for Adaptive Line Enhancing",Ph.D Thesis,Imperial College,1990

[8] N.DeClaris,"An Approximation Method with Rational Functions", Tech.Report,M.I.T.,1954

[9] S.Haykin,"Adaptive Filter Theory",PrenticeHall,1991

[10] W.H.Kautz,"Transient Synthesis in the Time Domain", IRE Trans.Cet.Theory,v1,1954 
[11] G.Davidson and D.Falconer, "Reduced Complexity Echo Cancellation using Orthonormal Functions",IREE Trans.Cet.Sys.,v38-1,1991

[12] P.Broome, "Discrete Orthonormal Sequences", J.A.Comp.Machinery,v12-2,1965

[13] X.Nie, D.Raghuramireddy and R.Unbehaven, "Orthonormal Expansion of Stable Rational Transfer Functions",Elec.Let., v27,No.16,1991

[14] A.Swami and J.M.Mendel,"Cumulant-Based Approach to the Harmonic Retrieval Problem", Proc.ICASSP-88,New York,1988

[15] V.F.Pisarenko, The Retrieval of Harmonics from a Covariance Function", Geophys. J.R. Astro. Soc., v33,1973

Figures

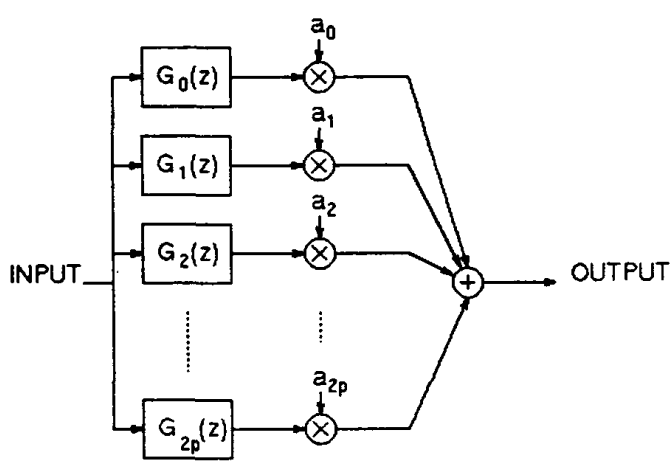

Figure 1. Orthogonal Block Fiter

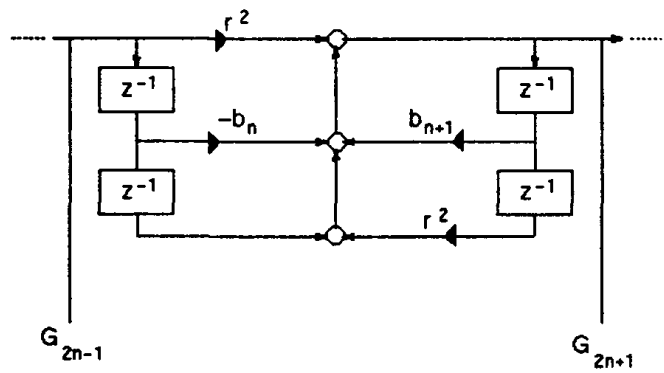

Figure 2. Real Orthgogonal Block Reallsation

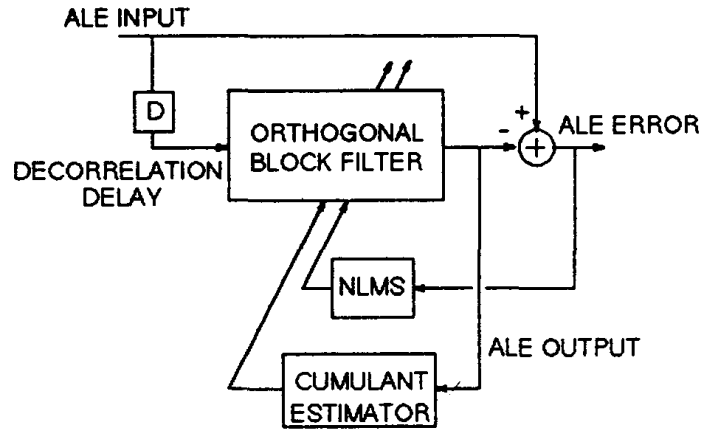

Figure 3. Orthogonal Block Adaptive Line Enhancer

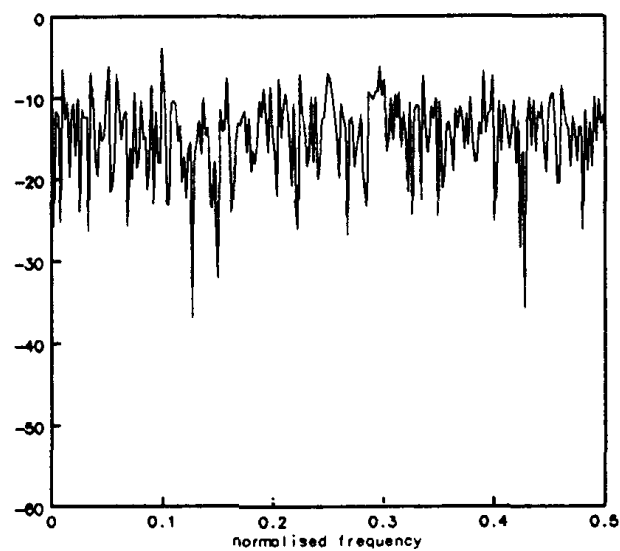

Figure 4. Power Spectrum of OBALE Input, -15dB SNR

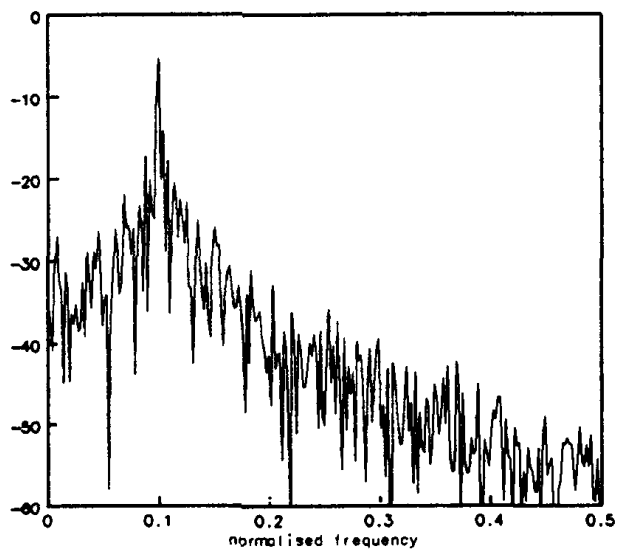

Figure 5. Power Spectrum of OBALE output 